\title{
Synthesis of thiophenyl thiazole based novel quinoxaline derivatives
}

\author{
Haresh G. Kathrotiya, Yogesh T. Naliapara* \\ Department of Chemistry, Saurashtra University, Rajkot-360005, Gujarat, India \\ *E-mail address: naliaparachem@yahoo.co.in
}

Keywords: Thiophenyl; Thiazole; Quinoxaline

\begin{abstract}
A new series of thiophenyl thiazole based novel quinoxaline derivatives $\mathbf{4 a - 4 t}$ have been synthesized by base catalysed condensation reaction. In which 6-substituted 2,3dichloroquinoxaline 1a and 4-(thiophen-2-yl)thiazol-2-amine $\mathbf{2 b}$ reacted in basic condition to afford intermediate $\mathbf{3 c}$ which reacts with various aromatic amine to form final compounds. Easy experimental procedure, high yield, and selectivity are the imperative features of this method. The identity of all the compounds has been established by ${ }^{1} \mathrm{H}$ NMR, ${ }^{13} \mathrm{C}$ NMR, FT-IR, and elemental analysis.
\end{abstract}

\section{INTRODUCTION}

A wide variety of pharmacological properties has been associated with quinoxaline derivatives. Quinoxaline also called benzopyrazine is a heterocyclic compound containing a ring complex made up of benzene ring and a pyrazine ring has been considered as a wonderful nucleus. Quinoxalines have extraordinary potential in pharmacological research [1] and practice. These are important components of several pharmacologically active compounds [2-8] and exhibit special and wider ranges of functions in biologically active compounds [9], electroluminescent materials [10], dyes [11] and anion sensors [12]. Although rarely describe in nature, synthetic quinoxaline derivatives showed variety of pharmaceutical activities encompassed major types of drug target families and effective in many clinical applications such as anti tumor agents [13], kinase inhibitors [14], HIV drugs [15], antibiotics [16], ion channel regulators [17] and anti protozoal agents [18].

Numerous methods are available for the synthesis of quinoxaline derivatives which involve condensation of 1,2-diamines with $\alpha$-diketones $[19,20], 1,4$-addition of 1,2-diamines to diazenylbutenes [21], cyclization-oxidation of phenacyl bromides [22, 23] and oxidative coupling of epoxides with ene-1,2-diamines [24], 2,3-Disubstituted quinoxalines have also been prepared via the Suzuki-Miyaura coupling reaction [25], condensation of $o$-phenylenediamines with 1,2dicarbonyl compounds in $\mathrm{MeOH} / \mathrm{AcOH}$ under microwave irradiation [26] and iodine catalyzed cyclocondensation of 1,2-dicarbonyl compounds with substituted $o$-phenylenediamines in DMSO [27] or $\mathrm{CH}_{3} \mathrm{CN}$ [28].

A variety of catalysts were tested in these reactions such as acetic acid [29], iodine [30], $\mathrm{CuSO}_{4} .5 \mathrm{H}_{2} \mathrm{O}$ [31], nickel nanoparticles [32], gallium(III)triflate [33], montmorillonite K-10 [34], ionic liquids [35], Nano- $\mathrm{TiO}_{2}$ [36], sulfated $\mathrm{TiO}_{2}$ [37], $\mathrm{Pd}(\mathrm{OAc})_{2}$ [38], $\mathrm{RuCl}_{2}-\left(\mathrm{PPh}_{3}\right)_{3}-2,2,6,6-$ tetramethylpiperidine 1-oxyl(TEMPO) [38], $\mathrm{MnO}_{2}$ [38], $\mathrm{Al}_{2} \mathrm{O}_{3}$ [39], zirconium(IV)-modified silica gel [40], nanocrystalline $\mathrm{CuO}$ [41], cerium(IV) ammonium nitrate [42], iron exchanged molybdophosphoric acid [43], silica-bonded S-sulfonic acid [44], and sulfamic acid/MeOH [45]. Here we report synthesis of novel thiophene thiazole based quinoxaline derivatives by chloro-amine condensation. The constitutions of all the products were confirmed using ${ }^{1} \mathrm{H} \mathrm{NMR},{ }^{13} \mathrm{C} N M R$, FTIR, and elemental analysis. 


\section{EXPERIMENTAL}

Required all reagents were obtained commercially. Solvents were purified and dried before being used. All melting points were taken in open capillaries and are uncorrected. Thin-layer chromatography (TLC, on aluminium plates precoated with silica gel, $60 \mathrm{~F}_{254}, 0.25 \mathrm{~mm}$ thickness) (Merck, Darmstadt, Germany) was used for monitoring the progress of all reactions, purity and homogeneity of the synthesized compounds; eluent-hexane:ethyl acetate: (4:6). UV radiation and/or iodine were used as the visualizing agents. Elemental analysis $(\% \mathrm{C}, \mathrm{H}, \mathrm{N})$ was carried out by Perkin-Elmer 2400 series-II elemental analyzer (Perkin-Elmer, USA) and all compounds are within $\pm 0.4 \%$ of theory specified. The IR spectra were recorded in $\mathrm{KBr}$ on a Perkin-Elmer Spectrum GX FT-IR Spectrophotometer (Perkin-Elmer, USA) and only the characteristic peaks are reported in $\mathrm{cm}^{-1} .{ }^{1} \mathrm{H}$ NMR and ${ }^{13} \mathrm{C}$ NMR spectra were recorded in DMSO- $d_{6}$ on a Bruker Avance $400 \mathrm{~F}(\mathrm{MHz})$ spectrometer (Bruker Scientific Corporation Ltd., Switzerland) using solvent peak as internal standard at $400 \mathrm{MHz}$ and $100 \mathrm{MHz}$ respectively. Chemical shifts are reported in parts per million (ppm). Mass spectra were scanned on a Shimadzu LCMS 2010 spectrometer (Shimadzu, Tokyo, Japan).

\subsection{Synthesis of the substituted 2,3-dichloroquinoxaline (1a)}

Substituted benzene-1,2-diamine $(5 \mathrm{mmol})$, diethyl oxalate $(5 \mathrm{mmol})$, piperidine $(1 \mathrm{ml})$, and ethanol $(10 \mathrm{ml})$ were charged in a $100 \mathrm{ml}$ round bottom flask with mechanical stirrer and condenser. The reaction mixture refluxed for $4 \mathrm{~h}$. After the completion of reaction (checked by TLC), the separated substituted quinoxaline-2,3(1H,4H)-dione was filtered and washed with ethanol and dried. Now thionyl chloride $(25 \mathrm{mmol})$ was added to a solution of substituted quinoxaline$2,3(1 H, 4 H)$-dione $(10 \mathrm{gm})$ in dry DCM $(50 \mathrm{~mL})$. Upon the addition of $1-2$ drop of DMF a mixture was heated at reflux for $1 \mathrm{~h}$. After the completion of reaction a mixture was washed with water followed by saturated $\mathrm{NaHCO}_{3}$ solution. The organic phase was dried over anhydrous $\mathrm{Na}_{2} \mathrm{SO}_{4}$ and solvent was removed under reduced pressure to afford analytically pure substituted 2,3dichloroquinoxaline.

\subsection{Synthesis of the substituted 3-chloro- $N$-(4-(thiophen-2-yl)thiazol-2-yl)quinoxalin-2- amine (3c)}

Substituted 2,3-dichloroquinoxaline $\mathbf{1 a}(5 \mathrm{mmol})$ and 4-(thiophen-2-yl)thiazol-2-amine $\mathbf{2 b}$ ( $5 \mathrm{mmol})$ were thoroughly mixed in DMF $(10 \mathrm{ml})$ and then dry $\mathrm{K}_{2} \mathrm{CO}_{3}(5 \mathrm{mmol})$ was added to it. The mixture was heated at $100{ }^{0} \mathrm{C} 3 \mathrm{~h}$. After the completion of reaction (checked by TLC), the solution was cooled to room temperature. The reaction mixture was poured in to chilled water. The separated precipitates were filtered, thoroughly washed well with water, dried, and recrystallized from ethanol to achive pure substituted 3-chloro- $N$-(4-(thiophen-2-yl)thiazol-2-yl)quinoxalin-2amine.

\subsection{General procedure for the synthesis of compounds 4(a-t)}

Substituted 3-chloro- $N$-(4-(thiophen-2-yl)thiazol-2-yl)quinoxalin-2-amine $3 \mathbf{c}(5 \mathrm{mmol})$ and various aromatic amines $(5 \mathrm{mmol})$ were thoroughly mixed in DMF $(10 \mathrm{ml})$ and then dry $\mathrm{K}_{2} \mathrm{CO}_{3}(5$ mmol) was added to it. The mixture was heated at $100{ }^{0} \mathrm{C}$ for $4-5 \mathrm{~h}$. After the completion of reaction (checked by TLC), the solution was cooled to room temperature. The reaction mixture was poured in to chilled water. The separated precipitates were filtered, thoroughly washed well with water, dried, and recrystallized from chloroform to obtain the pure compounds $\mathbf{4 a}-\mathbf{4 t}$. The physicochemical and spectroscopic characterization data of the synthesized compounds $\mathbf{4 a}-\mathbf{4 t}$ given below. 


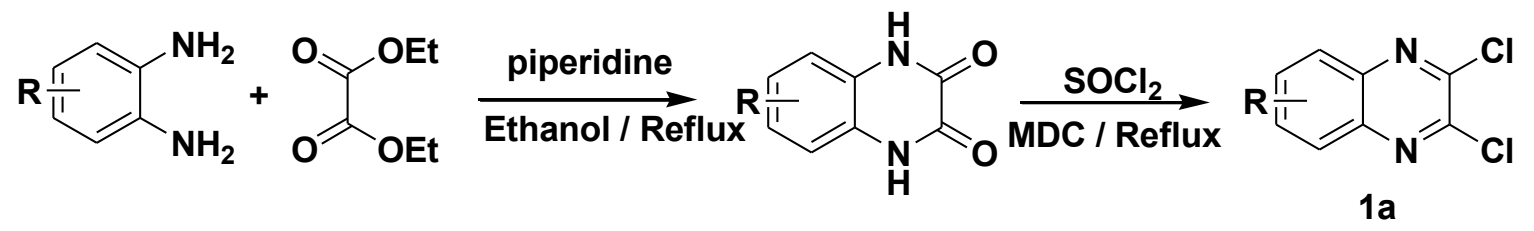

Where $\mathrm{R}=\mathrm{H}, \mathrm{CH}_{3}$

Scheme 1 Synthetic pathway for synthesis of $1 \mathrm{a}$

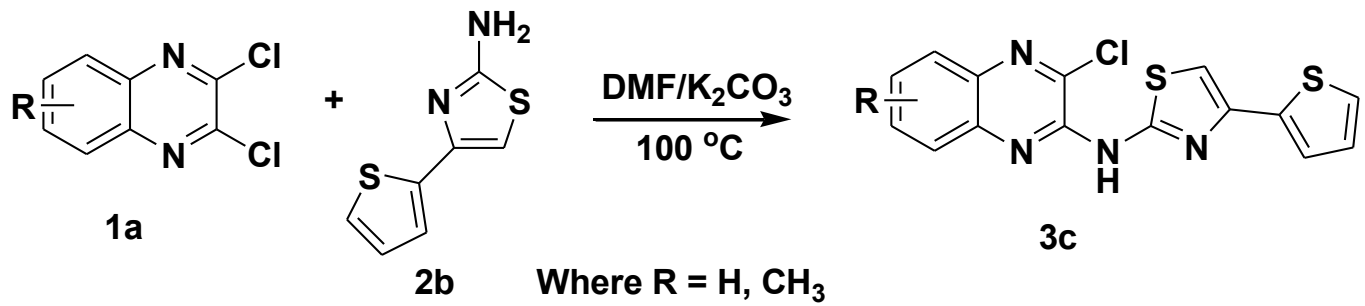

Scheme 2 Synthetic pathway for synthesis of $3 \mathrm{c}$

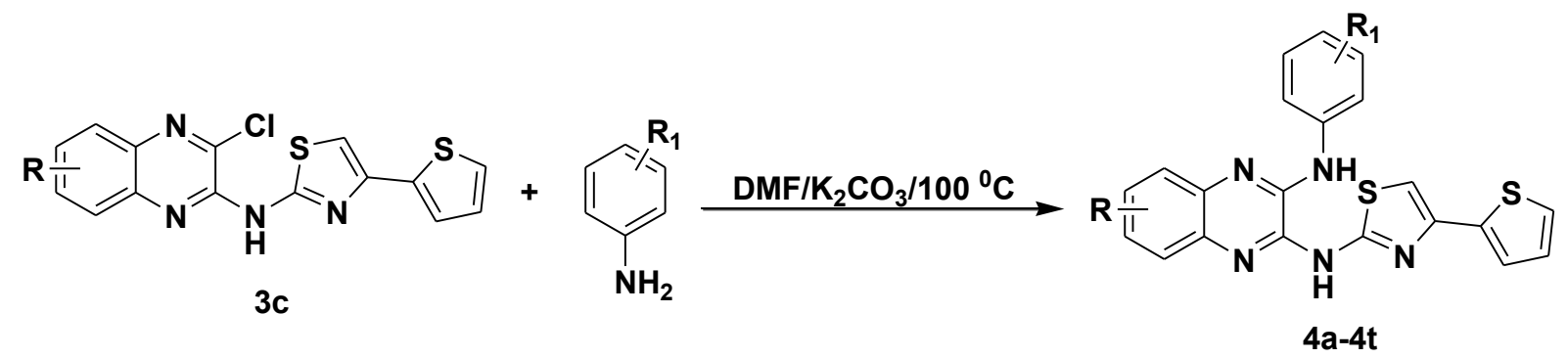

Where $\mathrm{R}_{1}=\mathrm{H}, \mathrm{CH}_{3}, \mathrm{OCH}_{3}, \mathrm{~F}, \mathrm{Cl}, \mathrm{Br}, \mathrm{NO}_{2}, \mathrm{OH}, \mathrm{NH}_{2}$

Scheme 3 Synthetic pathway for synthesis of thiophenyl thiazole based quinoxaline derivatives $4 \mathrm{a}-4 \mathrm{t}$

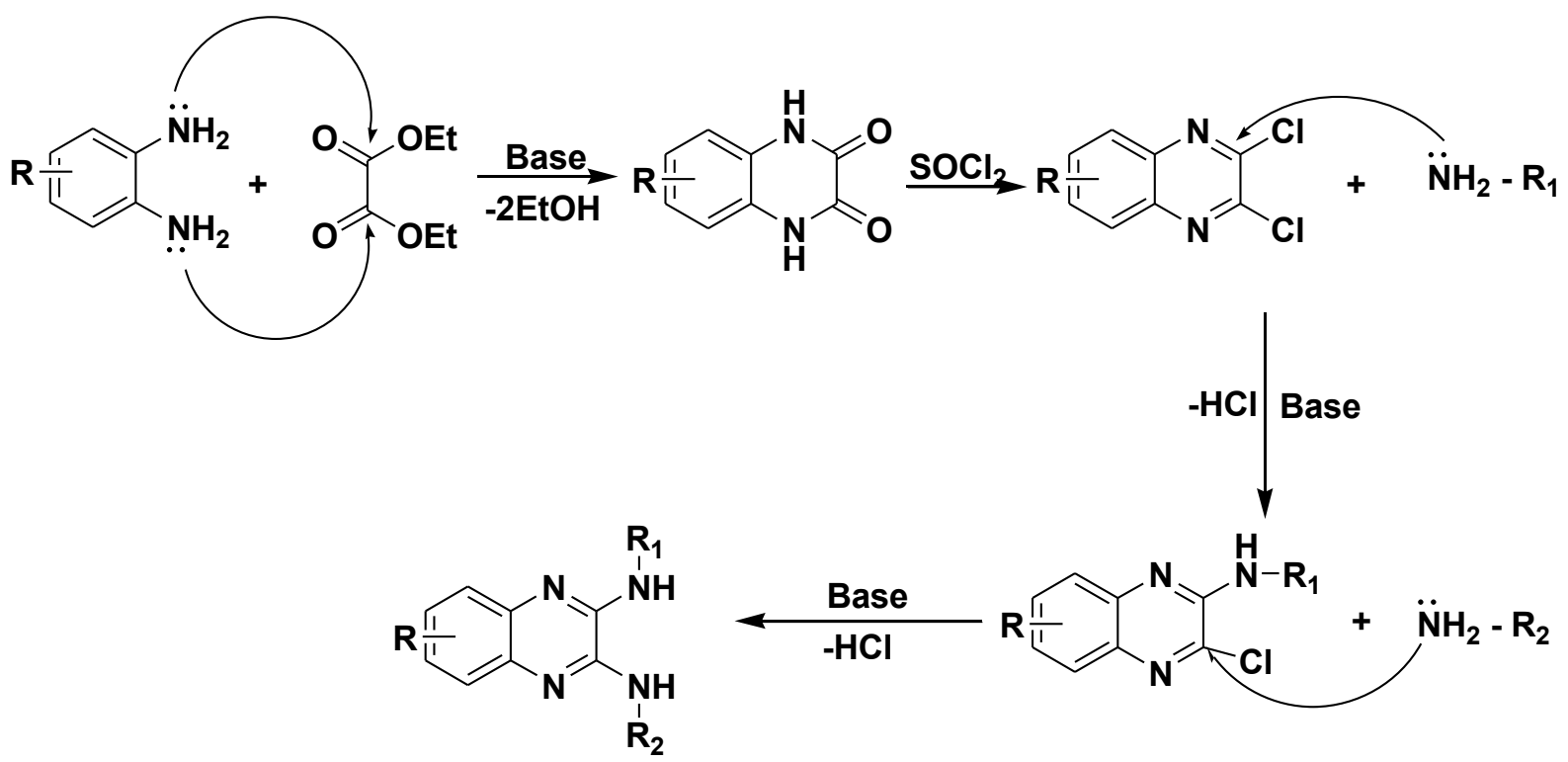

Where $\mathrm{R}=\mathrm{H}, \mathrm{CH}_{3}$

$\mathbf{R}_{1}=$ Heterocyles

$\mathbf{R}_{\mathbf{2}}=$ Aryl

Scheme 4 Mechanism for the synthesis of thiophenyl thiazole based quinoxaline derivative 
Table 1 Synthesis of quinoxaline derivatives $4 \mathrm{a}-4 \mathrm{t}$

\begin{tabular}{|c|c|c|c|c|c|}
\hline Entry & $\mathbf{R}$ & $\mathbf{R}_{\mathbf{1}}$ & $\mathbf{R T}(\mathbf{h .})$ & Yield \% & mp ${ }^{\mathbf{0}} \mathbf{C}$ \\
\hline $4 \mathrm{a}$ & $\mathrm{H}$ & $\mathrm{H}$ & 4.1 & 90 & $180-182$ \\
\hline $4 \mathrm{~b}$ & $\mathrm{H}$ & $4-\mathrm{CH}_{3}$ & 4 & 93 & $186-188$ \\
\hline $4 \mathrm{c}$ & $\mathrm{H}$ & $4-\mathrm{OCH}_{3}$ & 4.1 & 90 & $173-175$ \\
\hline $4 \mathrm{~d}$ & $\mathrm{H}$ & $4-\mathrm{F}$ & 4.3 & 88 & $162-164$ \\
\hline $4 \mathrm{e}$ & $\mathrm{H}$ & $4-\mathrm{Cl}$ & 4.4 & 88 & $190-192$ \\
\hline $4 \mathrm{f}$ & $\mathrm{H}$ & $4-\mathrm{Br}$ & 4.5 & 85 & $161-163$ \\
\hline $4 \mathrm{~g}$ & $\mathrm{H}$ & $4-\mathrm{OH}$ & 4.5 & 84 & $158-160$ \\
\hline $4 \mathrm{~h}$ & $\mathrm{H}$ & $4-\mathrm{NO}_{2}$ & 4.1 & 92 & $173-175$ \\
\hline $4 \mathrm{i}$ & $\mathrm{H}$ & $4-\mathrm{NH}_{2}$ & 4.6 & 80 & $166-168$ \\
\hline $4 \mathrm{j}$ & $\mathrm{H}$ & $3-\mathrm{CH}_{3}$ & 4.6 & 70 & $151-153$ \\
\hline $4 \mathrm{k}$ & $\mathrm{CH}_{3}$ & $\mathrm{H}$ & 4.3 & 89 & $130-132$ \\
\hline 41 & $\mathrm{CH}_{3}$ & $4-\mathrm{CH}_{3}$ & 4.1 & 90 & $142-148$ \\
\hline $4 \mathrm{~m}$ & $\mathrm{CH}_{3}$ & $4-\mathrm{OCH}_{3}$ & 4.2 & 91 & $136-138$ \\
\hline $4 \mathrm{n}$ & $\mathrm{CH}_{3}$ & $4-\mathrm{F}$ & 4.4 & 85 & $123-125$ \\
\hline $4 \mathrm{o}$ & $\mathrm{CH}_{3}$ & $4-\mathrm{Cl}_{3}$ & 4.5 & 84 & $120-122$ \\
\hline $4 \mathrm{p}$ & $\mathrm{CH}_{3}$ & $4-\mathrm{Br}_{4}$ & 4.5 & 84 & $136-138$ \\
\hline $4 \mathrm{q}$ & $\mathrm{CH}_{3}$ & $4-\mathrm{OH}_{3}$ & 4.6 & 86 & $127-129$ \\
\hline $4 \mathrm{r}$ & $\mathrm{CH}_{3}$ & $4-\mathrm{NO}_{2}$ & 4 & 90 & $119-121$ \\
\hline $4 \mathrm{~s}$ & $\mathrm{CH}_{3}$ & $4-\mathrm{NH}_{2}$ & 4.7 & 79 & $133-135$ \\
\hline $4 \mathrm{t}$ & $\mathrm{CH}_{3}$ & $3-\mathrm{CH}_{3}$ & 4.8 & 73 & $140-142$ \\
\hline & & & & & \\
\hline
\end{tabular}

The required 4-(thiophen-2-yl)thiazol-2-amine $\mathbf{2 b}$ was prepared by solid phase reaction according to literature procedure [46].

In this study, a series of quinoxaline derivatives $\mathbf{4 a}-\mathbf{4 t}$ have been synthesized by chloroamine condensation reaction of substituted 3-chloro- $N$-(4-(thiophen-2-yl)thiazol-2-yl)quinoxalin-2amine and various aromatic amines in DMF at $100{ }^{0} \mathrm{C}$ using $\mathrm{K}_{2} \mathrm{CO}_{3}$ as a base (scheme-3). According to the mechanism suggested in (scheme-4), the formation of the quinoxaline derivatives occurs via chloro-amine condensation in presence of $\mathrm{K}_{2} \mathrm{CO}_{3}$. Reaction of substituted benzene-1,2diamine and diethyl oxalate gave substituted quinoxaline-2,3(1H,4H)-dione by removel of two ethanol molecules. Then substituted quinoxaline-2,3(1H,4H)-dione treated by $\mathrm{SOCl}_{2}$ to obtaine substituted 2,3-dichloroquinoxaline. At last, nucleophilic attack of amine to the substituted 2,3dichloroquinoxaline moiety took place to afford quinoxaline derivatives $\mathbf{4 a}-\mathbf{4 t}$ (Scheme 4).

The structures of all the new synthesized compounds were established by ${ }^{1} \mathrm{H}$ NMR, ${ }^{13} \mathrm{C}$ NMR, FTIR, elemental analysis, and molecular weight of some selected compounds were confirmed by mass spectrometry. In ${ }^{1} \mathrm{H}$ NMR, (DMSO-d6) spectrum of compound $\mathbf{4 b}$ two singlet peak at d $10.41 \mathrm{ppm}$ and d $10.62 \mathrm{ppm}$ appeared for two $-\mathrm{NH}-$ protons. Aromatic protons of $\mathbf{4 b}$ resonate as multiplets at $\mathrm{d} 6.90-8.00 \mathrm{ppm}$. A singlet at $\mathrm{d} 2.80 \mathrm{ppm}$ stands for aromatic methyl protons. ${ }^{13} \mathrm{C}$ NMR of $\mathbf{4 b}$ exhibited a distinctive signal at $\mathrm{d} 27.12 \mathrm{ppm}$ for aromatic methyl. All the aromatic carbons of $\mathbf{4 b}$ showed signals at $\mathrm{d}$ 107.25-138.39 ppm in the ${ }^{13} \mathrm{C} N M R$ spectra. The IR spectrum of compound $4 \mathrm{~b}$ exhibited characteristic absorption band at 3,318 and 3,226 $\mathrm{cm}^{-1}$ for two $-\mathrm{NH}-$ and $3,026 \mathrm{~cm}^{-1}$ for aromatic $\mathrm{C}-\mathrm{H}$ stretching. Further, the structures of selected compounds $\mathbf{4 b}, \mathbf{4 c}$, and $\mathbf{4 k}$ were confirmed by its mass spectral studies. The mass spectra detected the expected molecular ion signals corresponding to respective molecular formula of synthesized compounds. Mass spectra of compound $\mathbf{4 b}$ gave molecular ion peak at $415.0(\mathrm{M}+1)$ corresponding to molecular formula $\mathrm{C}_{22} \mathrm{H}_{17} \mathrm{~N}_{5} \mathrm{~S}_{2}$. All spectroscopic data have been given in spectral data. 


\section{SPECTRAL DATA}

\section{$\boldsymbol{N}^{2}$-phenyl- $\boldsymbol{N}^{\mathbf{3}}$-(4-(thiophen-2-yl)thiazol-2-yl)quinoxaline-2,3-diamine (4a)}

yellow solid, yield 90\%, m.p. 180-182 C, IR (KBr, v, cm ${ }^{-1}$ ): 3325 (N-H Str.), 3220 (N-H Str.), 3024 (Ar C-H Str.), ${ }^{1} \mathrm{H}$ NMR (400 MHz, DMSO- $\left.d_{6}\right) \delta_{\mathrm{H}}(\mathrm{ppm}): \delta 6.520-8.022(\mathrm{~m}, 13 \mathrm{H}, \mathrm{Ar}-\mathrm{H}), 10.208(\mathrm{~s}$, $1 \mathrm{H}, \mathrm{NH}), 10.520(\mathrm{~s}, 1 \mathrm{H}, \mathrm{NH}) .{ }^{13} \mathrm{C}$ NMR $\left(100 \mathrm{MHz}, \mathrm{DMSO}-d_{6}\right) \delta_{\mathrm{C}}(\mathrm{ppm}): 107.12,108.25,109.00$, $110.11,112.32$, 113.00, 114.15, 116.00, 118.32, 119.00, 121.04, 122.32, 123.01, 125.18, 127.93, 128.56, 130.00, 132.35, 134.89, 138.25, 139.28(Ar-C). $\mathrm{MS}\left(\mathrm{M}^{+}\right):$401.08, Anal. Calcd. for $\mathrm{C}_{21} \mathrm{H}_{15} \mathrm{~N}_{5} \mathrm{~S}_{2}$ (401.51): C 62.82, H 3.77, N 17.44 Found: C 62.70, H 3.45, N 17.52\%.

\section{$\boldsymbol{N}^{\mathbf{2}}$-(4-(thiophen-2-yl)thiazol-2-yl)- $\boldsymbol{N}^{3}$-p-tolylquinoxaline-2,3-diamine (4b)}

yellow solid, yield 93\%, m.p. 186-188 C, IR (KBr, v, $\mathrm{cm}^{-1}$ ): 3318 (N-H Str.), 3216 (N-H Str.), 3026 (Ar C-H Str.), ${ }^{1} \mathrm{H}$ NMR (400 MHz, DMSO- $\left.d_{6}\right) \delta_{\mathrm{H}}(\mathrm{ppm}): \delta 2.800\left(\mathrm{~s}, 3 \mathrm{H}, \mathrm{CH}_{3}\right), 6.900-8.003(\mathrm{~m}$, $12 \mathrm{H}, \mathrm{Ar}-\mathrm{H}), 10.412(\mathrm{~s}, 1 \mathrm{H}, \mathrm{NH}), 10.620(\mathrm{~s}, 1 \mathrm{H}, \mathrm{NH}) .{ }^{13} \mathrm{C}$ NMR $\left(100 \mathrm{MHz}, \mathrm{DMSO}-d_{6}\right) \delta_{\mathrm{C}}(\mathrm{ppm})$ : $27.12\left(\mathrm{Ar}_{-} \mathrm{CH}_{3}\right), 107.25,108.44,109.77,110.59,111.30,112.60,114.60,115.10,118.20,119.18$, $121.40,122.36,123.22,124.27,126.73,128.42,130.65,131.65,134.50,137.33,138.39($ Ar-C). MS( $\left.\mathrm{M}^{+}\right)$: 415.09, Anal. Calcd. for $\mathrm{C}_{22} \mathrm{H}_{17} \mathrm{~N}_{5} \mathrm{~S}_{2}$ (415.53): C 63.59, H 4.12, N 16.85 Found: C 63.80, H 4.15, N 16.30\%.

\section{$\boldsymbol{N}^{2}$-(4-methoxyphenyl)- $\boldsymbol{N}^{3}$-(4-(thiophen-2-yl)thiazol-2-yl)quinoxaline-2,3-diamine (4c)}

yellow solid, yield 90\%, m.p. $173-175^{\circ} \mathrm{C}$, IR (KBr, v, $\mathrm{cm}^{-1}$ ): 3313 (N-H Str.), 3223 (N-H Str.), 3010 (Ar C-H Str.), ${ }^{1} \mathrm{H}$ NMR (400 MHz, DMSO-d $) \delta_{\mathrm{H}}(\mathrm{ppm}): \delta 3.725\left(\mathrm{~s}, 3 \mathrm{H}, \mathrm{OCH}_{3}\right), 7.120-8.321(\mathrm{~m}$, $12 \mathrm{H}, \mathrm{Ar}-\mathrm{H}), 10.133(\mathrm{~s}, 1 \mathrm{H}, \mathrm{NH}), 10.236(\mathrm{~s}, 1 \mathrm{H}, \mathrm{NH}) .{ }^{13} \mathrm{C}$ NMR $\left(100 \mathrm{MHz}, \mathrm{DMSO}-d_{6}\right) \delta_{\mathrm{C}}(\mathrm{ppm})$ : $58.00\left(\mathrm{OCH}_{3}\right), 106.13,107.20,108.11,110.54,112.36,113.28,114.20,116.72,118.33,120.00$, $121.22,122.14,123.31,124.18,125.73,128.64,130.30,132.12,133.56,136.30,137.98$ (Ar-C). $\mathrm{MS}\left(\mathrm{M}^{+}\right)$: 431.09, Anal. Calcd. for $\mathrm{C}_{22} \mathrm{H}_{17} \mathrm{~N}_{5} \mathrm{OS}_{2}$ (431.53): C 61.23, H 3.97, N 16.23 Found: C 61.12, H 4.02, N 16.70\%.

\section{$\boldsymbol{N}^{\mathbf{2}}$-(4-fluorophenyl)- $\boldsymbol{N}^{\mathbf{3}}$-(4-(thiophen-2-yl)thiazol-2-yl)quinoxaline-2,3-diamine (4d)}

yellow solid, yield 88\%, m.p. 162-164 C, IR (KBr, v, $\mathrm{cm}^{-1}$ ): 3318 (N-H Str.), 3216 (N-H Str.), 3028 (Ar C-H Str.), ${ }^{1} \mathrm{H}$ NMR (400 MHz, DMSO-d $) \delta_{\mathrm{H}}(\mathrm{ppm}): \delta 6.998-7.846(\mathrm{~m}, 12 \mathrm{H}, \mathrm{Ar}-\mathrm{H}), 10.648(\mathrm{~s}$, $1 \mathrm{H}, \mathrm{NH}), 10.715(\mathrm{~s}, 1 \mathrm{H}, \mathrm{NH}) .{ }^{13} \mathrm{C}$ NMR $\left(100 \mathrm{MHz}, \mathrm{DMSO}-d_{6}\right) \delta_{\mathrm{C}}(\mathrm{ppm}): 108.15,109.16,110.23$, $112.00,113.66,114.03,115.28,116.30,118.00,119.36,120.22,122.00,123.30,124.21,126.20$, 127.36, 128.00, 129.60, 131.02, 133.06, 135.24(Ar-C). $\mathrm{MS}\left(\mathrm{M}^{+}\right)$: 419.07, Anal. Calcd. for $\mathrm{C}_{21} \mathrm{H}_{14} \mathrm{FN}_{5} \mathrm{~S}_{2}$ (419.5): C 60.13, H 3.36, N 16.69 Found: C 60.35, H 3.42, N 16.77\%.

\section{$\boldsymbol{N}^{\mathbf{2}}$-(4-chlorophenyl)- $\boldsymbol{N}^{\mathbf{3}}$-(4-(thiophen-2-yl)thiazol-2-yl)quinoxaline-2,3-diamine (4e)}

yellow solid, yield 88\%, m.p. 190-192 C, IR (KBr, v, cm ${ }^{-1}$ ): 3344 (N-H Str.), 3248 (N-H Str.), 3001 (Ar C-H Str.), ${ }^{1} \mathrm{H}$ NMR (400 MHz, DMSO- $\left.d_{6}\right) \delta_{\mathrm{H}}(\mathrm{ppm}): \delta$ 7.120-8.300 (m, 12H, Ar-H), $10.123(\mathrm{~s}$, $1 \mathrm{H}, \mathrm{NH}), 10.236(\mathrm{~s}, 1 \mathrm{H}, \mathrm{NH}) .{ }^{13} \mathrm{C} \mathrm{NMR}\left(100 \mathrm{MHz}, \mathrm{DMSO}-d_{6}\right) \delta_{\mathrm{C}}(\mathrm{ppm}): 108.63,109.56,111.13$, $112.25,113.78,114.55,115.35,116.65,118.12,119.93,120.88,122.00,122.90,123.11,125.10$, 126.33, 128.01, 129.45, 131.20, 132.15, 134.14 (Ar-C). $\mathrm{MS}\left(\mathrm{M}^{+}\right)$: 435.04, Anal. Calcd. for $\mathrm{C}_{21} \mathrm{H}_{14} \mathrm{ClN}_{5} \mathrm{~S}_{2}$ (435.95): C 57.86, H 3.24, N 16.06 Found: C 57.50, H 3.36, N 16.20\%.

\section{$\boldsymbol{N}^{\mathbf{2}}$-(4-bromophenyl)- $\boldsymbol{N}^{\mathbf{3}}$-(4-(thiophen-2-yl)thiazol-2-yl)quinoxaline-2,3-diamine (4f)}

yellow solid, yield 85\%, m.p. 161-163 ${ }^{\circ} \mathrm{C}$, IR (KBr, $v, \mathrm{~cm}^{-1}$ ): 3312 (N-H Str.), 3233 (N-H Str.), 3016 (Ar C-H Str.), ${ }^{1} \mathrm{H}$ NMR (400 MHz, DMSO- $\left.d_{6}\right) \delta_{\mathrm{H}}(\mathrm{ppm}): \delta 6.888-7.894(\mathrm{~m}, 12 \mathrm{H}, \mathrm{Ar}-\mathrm{H}), 10.325$ (s, $1 \mathrm{H}, \mathrm{NH}), 10.423(\mathrm{~s}, 1 \mathrm{H}, \mathrm{NH}) .{ }^{13} \mathrm{C}$ NMR $\left(100 \mathrm{MHz}, \mathrm{DMSO}-d_{6}\right) \delta_{\mathrm{C}}(\mathrm{ppm}): 106.32,108.22,110.21$, $111.20,130.00,114.65,115.20,116.70,118.01,119.20,121.00,122.36,1124.77,125.88,127.80$, 129.36, 132.20, 133.54, 135.10, 137.11, 139.18(Ar-C). $\mathrm{MS}\left(\mathrm{M}^{+}\right)$: 478.99, Anal. Calcd. for $\mathrm{C}_{21} \mathrm{H}_{14} \mathrm{BrN}_{5} \mathrm{~S}_{2}$ (480.40): C 60.29, H 2.94, N 14.58 Found: C 60.43, H 3.03, N 14.70\%. 
4-(2-(4-(thiophen-2-yl)thiazol-2-ylamino)quinoxalin-3-ylamino)phenol (4g)

yellow solid, yield 84\%, m.p. 158-160 C, IR (KBr, v, $\left.\mathrm{cm}^{-1}\right)$ : 3300 (N-H Str.), 3211 (N-H Str.), 3040 (Ar C-H Str.), ${ }^{1} \mathrm{H}$ NMR (400 MHz, DMSO- $\left.d_{6}\right) \delta_{\mathrm{H}}(\mathrm{ppm}): \delta 4.856(\mathrm{~s}, 1 \mathrm{H}, \mathrm{OH}), 7.125-8.136(\mathrm{~m}$, $12 \mathrm{H}, \mathrm{Ar}-\mathrm{H}), 10.236(\mathrm{~s}, 1 \mathrm{H}, \mathrm{NH}), 10.312(\mathrm{~s}, 1 \mathrm{H}, \mathrm{NH}) .{ }^{13} \mathrm{C}$ NMR $\left(100 \mathrm{MHz}, \mathrm{DMSO}-d_{6}\right) \delta_{\mathrm{C}}(\mathrm{ppm})$ : 105.32 , 107.12, 109.26, 110.32, 112.60, 114.75, 115.50, 116.83, 118.41, 119.33, 121.17, 122.84, 124.37, 125.22, 126.30, 129.39, 131.22, 133.66, 134.12, 135.15, 138.17(Ar-C). MS( $\left.\mathrm{M}^{+}\right): 417.07$, Anal. Calcd. for $\mathrm{C}_{21} \mathrm{H}_{15} \mathrm{~N}_{5} \mathrm{OS}_{2}$ (417.51): $\mathrm{C} 60.41, \mathrm{H} 3.62, \mathrm{~N} 16.77$ Found: $\mathrm{C} 60.58, \mathrm{H} \mathrm{3.70,} \mathrm{N}$ $16.17 \%$.

$\boldsymbol{N}^{\mathbf{2}}$-(4-nitrophenyl)- $\boldsymbol{N}^{\mathbf{3}}$-(4-(thiophen-2-yl)thiazol-2-yl)quinoxaline-2,3-diamine (4h)

yellow solid, yield $92 \%$, m.p. $173-175^{\circ} \mathrm{C}$, IR (KBr, v, $\mathrm{cm}^{-1}$ ): 3327(N-H Str.), 3241 (N-H Str.), 3048 (Ar C-H Str.), ${ }^{1} \mathrm{H}$ NMR (400 MHz, DMSO- $\left.d_{6}\right) \delta_{\mathrm{H}}$ (ppm): $\delta$ 7.201-8.320 (m, 12H, Ar-H), 10.336 (s, $1 \mathrm{H}, \mathrm{NH}), 10.408(\mathrm{~s}, 1 \mathrm{H}, \mathrm{NH}) .{ }^{13} \mathrm{C}$ NMR $\left(100 \mathrm{MHz}, \mathrm{DMSO}-d_{6}\right) \delta_{\mathrm{C}}(\mathrm{ppm}): 107.88,108.90,110.35$, $111.37,112.98,114.40,115.26,116.78,118.22$, 119.35, 120.45, 122.55, 124.63, 125.72, 128.10,

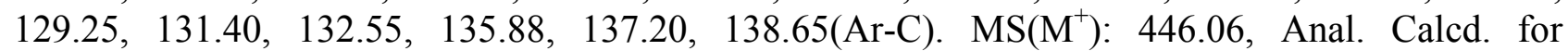
$\mathrm{C}_{21} \mathrm{H}_{14} \mathrm{~N}_{6} \mathrm{O}_{2} \mathrm{~S}_{2}$ (446.50): C 56.49, H 3.16, N 18.82 Found: C 56.30, H 3.33, N 18.62\%

$\boldsymbol{N}^{\mathbf{2}}$-(4-aminophenyl)- $\boldsymbol{N}^{3}$-(4-(thiophen-2-yl)thiazol-2-yl)quinoxaline-2,3-diamine (4i)

yellow solid, yield 80\%, m.p. 166-168 C, IR (KBr, v, $\mathrm{cm}^{-1}$ ): 3326 (N-H Str.), 3211 (N-H Str.), 3014 (Ar C-H Str.), ${ }^{1} \mathrm{H}$ NMR (400 MHz, DMSO-d $d_{6} \delta_{\mathrm{H}}(\mathrm{ppm}): \delta$ 7.200-8.365 (m, 12H, Ar-H), $10.123(\mathrm{~s}$, $1 \mathrm{H}, \mathrm{NH}), 10.220(\mathrm{~s}, 1 \mathrm{H}, \mathrm{NH}), 10.700\left(\mathrm{~s}, 2 \mathrm{H}, \mathrm{NH}_{2}\right) .{ }^{13} \mathrm{C}$ NMR $\left(100 \mathrm{MHz}, \mathrm{DMSO}-d_{6}\right) \delta_{\mathrm{C}}(\mathrm{ppm})$ : $109.31,111.00,112.48,113.54,115.24,116.44,117.41,119.05,120.33,122.27,124.50,126.40$, 128.73, 130.52, 132.33, 135.24, 137.88, 139.61, 142.01, 143.98, 145.00(Ar-C). MS(M $\left.{ }^{+}\right): 416.09$, Anal. Calcd. for $\mathrm{C}_{21} \mathrm{H}_{16} \mathrm{~N}_{6} \mathrm{~S}_{2}$ (416.52): $\mathrm{C} 60.55, \mathrm{H} \mathrm{3.87,} \mathrm{N} 20.18$ Found: $\mathrm{C} 60.43, \mathrm{H} \mathrm{3.70,} \mathrm{N}$ $20.50 \%$.

\section{$\boldsymbol{N}^{\mathbf{2}}$-(4-(thiophen-2-yl)thiazol-2-yl)- $\boldsymbol{N}^{\mathbf{3}}$-m-tolylquinoxaline-2,3-diamine (4j)}

yellow solid, yield 70\%, m.p. 151-153 C, IR (KBr, v, $\mathrm{cm}^{-1}$ ): 3290 (N-H Str.), 3216 (N-H Str.), 3015 (Ar C-H Str.), ${ }^{1} \mathrm{H}$ NMR (400 MHz, DMSO- $\left.d_{6}\right) \delta_{\mathrm{H}}(\mathrm{ppm}): \delta 2.563$ (s, 3H, $\left.\mathrm{CH}_{3}\right), 6.990-7.800(\mathrm{~m}$, 12H, Ar-H), $10.300(\mathrm{~s}, 1 \mathrm{H}, \mathrm{NH}), 10.412(\mathrm{~s}, 1 \mathrm{H}, \mathrm{NH}) .{ }^{13} \mathrm{C}$ NMR $\left(100 \mathrm{MHz}, \mathrm{DMSO}-d_{6}\right) \delta_{\mathrm{C}}(\mathrm{ppm})$ : $26.47\left(\mathrm{Ar}_{-} \mathrm{CH}_{3}\right), 109.42,111.56,112.53,114.44,115.36,116.28,117.91,118.06,120.13,122.57$, $124.33,125.30,128.66,130.14,132.20,135.54,136.78,138.71,141.25,142.16,144.01(\mathrm{Ar}-\mathrm{C})$. $\mathrm{MS}\left(\mathrm{M}^{+}\right)$: 415.09, Anal. Calcd. for $\mathrm{C}_{22} \mathrm{H}_{17} \mathrm{~N}_{5} \mathrm{~S}_{2}$ (415.53): C 63.59, H 4.12, N 16.85 Found: C 63.80, H 4.22, N $16.33 \%$.

\section{6-methyl- $\mathrm{N}^{3}$-phenyl- $\mathrm{N}^{2}$-(4-(thiophen-2-yl)thiazol-2-yl)quinoxaline-2,3-diamine (4k)}

yellow solid, yield 89\%, m.p. 130-132 C, IR (KBr, v, $\mathrm{cm}^{-1}$ ): 3280 (N-H Str.), 3225 (N-H Str.), 3013 (Ar C-H Str.), ${ }^{1} \mathrm{H}$ NMR (400 MHz, DMSO-d $\left.d_{6}\right) \delta_{\mathrm{H}}(\mathrm{ppm}): \delta 2.520\left(\mathrm{~s}, 3 \mathrm{H}, \mathrm{Ar}-\mathrm{CH}_{3}\right), 6.800-7.920(\mathrm{~m}$, 12H, Ar-H), $10.302(\mathrm{~s}, 1 \mathrm{H}, \mathrm{NH}), 10.613(\mathrm{~s}, 1 \mathrm{H}, \mathrm{NH}) .{ }^{13} \mathrm{C}$ NMR $\left(100 \mathrm{MHz}, \mathrm{DMSO}-d_{6}\right) \delta_{\mathrm{C}}(\mathrm{ppm})$ : $23.16\left(\mathrm{Ar}_{-} \mathrm{CH}_{3}\right), 24.99\left(\mathrm{Ar}-\mathrm{CH}_{3}\right), 108.20,109.36,112.30,113.00,114.06,115.32,117.18,119.28$, $121.33,123.96,126.50,128.00,129.23,132.20,133.00,135.60,137.88,139.00,140.12,142.00$, 143.16(Ar-C). $\mathrm{MS}\left(\mathrm{M}^{+}\right)$: 415.09, Anal. Calcd. for $\mathrm{C}_{22} \mathrm{H}_{17} \mathrm{~N}_{5} \mathrm{~S}_{2}$ (415.53): C 63.59, H 4.12, N 16.85 Found: C 63.44, H 4.17, N 16.52\%.

\section{6-methyl- $\mathrm{N}^{2}$-(4-(thiophen-2-yl)thiazol-2-yl)- $\mathrm{N}^{3}$-p-tolylquinoxaline-2,3-diamine (4I)}

yellow solid, yield 90\%, m.p. 142-148 C, IR (KBr, v, $\mathrm{cm}^{-1}$ ): 3318 (N-H Str.), 3250 (N-H Str.), 3030 (Ar C-H Str.), ${ }^{1} \mathrm{H}$ NMR (400 MHz, DMSO- $\left.d_{6}\right) \delta_{\mathrm{H}}(\mathrm{ppm}): \delta 2.302$ (s, 3H, Ar-CH $\left.\mathrm{CH}_{3}\right), 2.415$ (s, 3H, Ar$\left.\mathrm{CH}_{3}\right), 7.120-8.100(\mathrm{~m}, 11 \mathrm{H}, \mathrm{Ar}-\mathrm{H}), 10.349(\mathrm{~s}, 1 \mathrm{H}, \mathrm{NH}), 10.500(\mathrm{~s}, 1 \mathrm{H}, \mathrm{NH}) .{ }^{13} \mathrm{C}$ NMR $(100 \mathrm{MHz}$, DMSO- $\left.d_{6}\right) \delta_{\mathrm{C}}(\mathrm{ppm}): 26.90\left(\mathrm{Ar}-\mathrm{CH}_{3}\right), 57.36\left(\mathrm{OCH}_{3}\right), 107.10,108.56,110.20,112.01,113.05$, $115.36,117.25,119.32,121.44,123.24,126.30,128.19,129.52,132.21,133.36,134.10,135.89$, 138.60, 140.35, 141.12, 143.96(Ar-C). $M S\left(M^{+}\right)$: 429.11, Anal. Calcd. for $\mathrm{C}_{23} \mathrm{H}_{19} \mathrm{~N}_{5} \mathrm{~S}_{2}$ (429.56): C 64.31, H 4.46, N 16.30 Found: C 64.42, H 4.70, N 16.48\%. 
$N^{3}$-(4-methoxyphenyl)-6-methyl- $N^{2}$-(4-(thiophen-2-yl)thiazol-2-yl)quinoxaline-2,3-diamine

(4m)

yellow solid, yield 91\%, m.p. 136-138 ${ }^{\circ} \mathrm{C}$, IR (KBr, $v, \mathrm{~cm}^{-1}$ ): 3344 (N-H Str.), 3223 (N-H Str.), 3012 (Ar C-H Str.), ${ }^{1} \mathrm{H}$ NMR (400 MHz, DMSO- $\left.d_{6}\right) \delta_{\mathrm{H}}(\mathrm{ppm}): \delta 2.301\left(\mathrm{~s}, 3 \mathrm{H}, \mathrm{Ar}-\mathrm{CH}_{3}\right), 3.412(\mathrm{~s}, 3 \mathrm{H}$, $\left.\mathrm{OCH}_{3}\right), 6.921-7.900(\mathrm{~m}, 11 \mathrm{H}, \mathrm{Ar}-\mathrm{H}), 10.256(\mathrm{~s}, 1 \mathrm{H}, \mathrm{NH}), 10.562(\mathrm{~s}, 1 \mathrm{H}, \mathrm{NH}),{ }^{13} \mathrm{C}$ NMR $(100$ MHz, DMSO- $\left.d_{6}\right) \delta_{\mathrm{C}}(\mathrm{ppm}): 24.40\left(\mathrm{Ar}-\mathrm{CH}_{3}\right), 106.22,109.35,112.18,113.23,114.49,115.12$, $117.33,119.91,121.25,123.62,126.61,127.10,128.20,131.40,133.15,135.45,137.18,139.24$, 140.16, 141.17, 142.15(Ar-C). $\mathrm{MS}\left(\mathrm{M}^{+}\right)$: 445.10, Anal. Calcd. for $\mathrm{C}_{23} \mathrm{H}_{19} \mathrm{~N}_{5} \mathrm{OS}_{2}$ (445.56): $\mathrm{C} 62.00$, H 4.30, N 15.72 Found: C 62.20, H 4.00, N 15.80\%.

$\boldsymbol{N}^{3}$-(4-fluorophenyl)-6-methyl- $\boldsymbol{N}^{\mathbf{2}}$-(4-(thiophen-2-yl)thiazol-2-yl)quinoxaline-2,3-diamine (4n) yellow solid, yield 85\%, m.p. $123-125^{\circ} \mathrm{C}$, IR (KBr, v, $\mathrm{cm}^{-1}$ ): 3298 (N-H Str.), 3314 (N-H Str.), 3001 (Ar C-H Str.), ${ }^{1} \mathrm{H}$ NMR (400 MHz, DMSO- $\left.d_{6}\right) \delta_{\mathrm{H}}(\mathrm{ppm}): \delta 2.600\left(\mathrm{~s}, 3 \mathrm{H}, \mathrm{Ar}-\mathrm{CH}_{3}\right), 7.100-8.326(\mathrm{~m}$, $11 \mathrm{H}, \mathrm{Ar}-\mathrm{H}), 10.112(\mathrm{~s}, 1 \mathrm{H}, \mathrm{NH}), 10.236(\mathrm{~s}, 1 \mathrm{H}, \mathrm{NH}){ }^{13} \mathrm{C}$ NMR $\left(100 \mathrm{MHz}, \mathrm{DMSO}-d_{6}\right) \delta_{\mathrm{C}}(\mathrm{ppm}):$ $27.00\left(\mathrm{Ar}_{-} \mathrm{CH}_{3}\right), 110.13,112.05,114.31,115.06,116.28,117.00,119.27,121.04,123.25,125.37$, 128.61, 130.66, 132, 05, 133.90, 135.22, 137.18, 139.14, 142.21, 143.02, 144.03, 146.10(Ar-C). $\mathrm{MS}\left(\mathrm{M}^{+}\right)$: 433.08, Anal. Calcd. for $\mathrm{C}_{22} \mathrm{H}_{16} \mathrm{FN}_{5} \mathrm{~S}_{2}$ (433.52): C 60.95, H 3.72, N 16.15 Found: C 60.80, H 3.90, N 16.02\%.

$N^{3}$-(4-chlorophenyl)-6-methyl- $\boldsymbol{N}^{2}$-(4-(thiophen-2-yl)thiazol-2-yl)quinoxaline-2,3-diamine (4o) yellow solid, yield 84\%, m.p. 120-122 C, IR (KBr, v, $\mathrm{cm}^{-1}$ ): 3294 (N-H Str.), 3246 (N-H Str.), 3053 (Ar C-H Str.), ${ }^{1} \mathrm{H}$ NMR (400 MHz, DMSO-d $\left.d_{6}\right) \delta_{\mathrm{H}}(\mathrm{ppm}): \delta 2.315\left(\mathrm{~s}, 3 \mathrm{H}, \mathrm{Ar}-\mathrm{CH}_{3}\right), 6.895-7.888(\mathrm{~m}$, $11 \mathrm{H}, \mathrm{Ar}-\mathrm{H}), 10.259(\mathrm{~s}, 1 \mathrm{H}, \mathrm{NH}), 10.400(\mathrm{~s}, 1 \mathrm{H}, \mathrm{NH}) .{ }^{13} \mathrm{C}$ NMR $\left(100 \mathrm{MHz}, \mathrm{DMSO}-d_{6}\right) \delta_{\mathrm{C}}(\mathrm{ppm})$ : $26.59\left(\mathrm{Ar}_{-} \mathrm{CH}_{3}\right), 111.15,112.35,114.51,115.82,116.34,117.36,119.61,121.32,123.44,124.17$, $127.51,130.39,132.05,133.10,135.82,137.98,139.22,142.78,143.25,143.90,145.10(\operatorname{Ar}-\mathrm{C})$. $\mathrm{MS}\left(\mathrm{M}^{+}\right)$: 449.05, Anal. Calcd. for $\mathrm{C}_{22} \mathrm{H}_{16} \mathrm{ClN}_{5} \mathrm{~S}_{2}$ (449.98): C 58.72, H 3.58, N 15.56 Found: C $58.78, \mathrm{H} 3.72, \mathrm{~N} 15.40 \%$.

$\boldsymbol{N}^{\mathbf{3}}$-(4-bromophenyl)-6-methyl- $\boldsymbol{N}^{\mathbf{2}}$-(4-(thiophen-2-yl)thiazol-2-yl)quinoxaline-2,3-diamine (4p) yellow solid, yield $84 \%$, m.p. $136-138^{\circ} \mathrm{C}$, IR (KBr, v, $\mathrm{cm}^{-1}$ ): 3308 (N-H Str.), 3256 (N-H Str.), 3016 (Ar C-H Str.), ${ }^{1} \mathrm{H}$ NMR (400 MHz, DMSO- $\left.d_{6}\right) \delta_{\mathrm{H}}(\mathrm{ppm}): \delta 2.515\left(\mathrm{~s}, 3 \mathrm{H}, \mathrm{Ar}-\mathrm{CH}_{3}\right), 7.120-8.000(\mathrm{~m}$, $11 \mathrm{H}, \mathrm{Ar}-\mathrm{H}), 10.369(\mathrm{~s}, 1 \mathrm{H}, \mathrm{NH}), 10.546(\mathrm{~s}, 1 \mathrm{H}, \mathrm{NH}) .{ }^{13} \mathrm{C}$ NMR $\left(100 \mathrm{MHz}, \mathrm{DMSO}-d_{6}\right) \delta_{\mathrm{C}}(\mathrm{ppm})$ : $23.96\left(\mathrm{Ar}_{-} \mathrm{CH}_{3}\right), 107.43,108.56,110.61,112.34,114.04,116.38,117.20,119.21,120.64,122.07$, $124.38,126.34,127.09,129.87,131.25,133.44,135.64,137.28,139.24,141.27,142.69(\mathrm{Ar}-\mathrm{C})$. $\mathrm{MS}\left(\mathrm{M}^{+}\right)$: 493.00, Anal. Calcd. for $\mathrm{C}_{22} \mathrm{H}_{16} \mathrm{BrN}_{5} \mathrm{~S}_{2}$ (494.43): C 53.44, H 3.26, N 14.16 Found: C 53.20, H 3.35, N 14.33\%.

4-(2-(4-(thiophen-2-yl)thiazol-2-ylamino)-6-methylquinoxalin-3-ylamino)phenol (4q)

yellow solid, yield $86 \%$, m.p. $127-129^{\circ} \mathrm{C}$, IR (KBr, v, cm ${ }^{-1}$ ): 3326 (N-H Str.), 3247 (N-H Str.), 3018 (Ar C-H Str.), ${ }^{1} \mathrm{H}$ NMR (400 MHz, DMSO-d $\left.{ }_{6}\right) \delta_{\mathrm{H}}(\mathrm{ppm}): \delta 2.125\left(\mathrm{~s}, 3 \mathrm{H}, \mathrm{Ar}_{-} \mathrm{CH}_{3}\right), 4.800(\mathrm{~s}, 1 \mathrm{H}$, $\mathrm{OH}), 7.084-8.125(\mathrm{~m}, 11 \mathrm{H}, \mathrm{Ar}-\mathrm{H}), 10.365(\mathrm{~s}, 1 \mathrm{H}, \mathrm{NH}), 10.654(\mathrm{~s}, 1 \mathrm{H}, \mathrm{NH}) .{ }^{13} \mathrm{C} \mathrm{NMR}(100 \mathrm{MHz}$, DMSO- $\left.d_{6}\right) \delta_{\mathrm{C}}(\mathrm{ppm}): 25.64\left(\mathrm{Ar}-\mathrm{CH}_{3}\right), 107.66,109.46,110.51,112.24,113.64,115.19,117.21$, 118.61, 120.55, 122.97, 124.65, 125.14, 127.98, 129.88, 131.15, 133.24, 135.24, 133.18, 136.04, 139.37, 141.68(Ar-C). $\mathrm{MS}\left(\mathrm{M}^{+}\right)$: 431.09, Anal. Calcd. for $\mathrm{C}_{22} \mathrm{H}_{17} \mathrm{~N}_{5} \mathrm{OS}_{2}$ (431.53): C 61.23, H 3.97, N 16.23 Found: C 61.45, H 4.07, N 16.10\%.

6-methyl- $\boldsymbol{N}^{\mathbf{3}}$-(4-nitrophenyl)- $\boldsymbol{N}^{\mathbf{2}}$-(4-(thiophen-2-yl)thiazol-2-yl)quinoxaline-2,3-diamine (4r) yellow solid, yield 90\%, m.p. 119-121 ${ }^{\circ} \mathrm{C}$, IR (KBr, v, $\mathrm{cm}^{-1}$ ): 3312 (N-H Str.), 3216 (N-H Str.), 3017 (Ar C-H Str.), ${ }^{1} \mathrm{H}$ NMR (400 MHz, DMSO-d $d_{6} \delta_{\mathrm{H}}(\mathrm{ppm}): \delta 2.312\left(\mathrm{~s}, 3 \mathrm{H}, \mathrm{Ar}-\mathrm{CH}_{3}\right), 6.985-8.045(\mathrm{~m}$, $11 \mathrm{H}, \mathrm{Ar}-\mathrm{H}), 10.100(\mathrm{~s}, 1 \mathrm{H}, \mathrm{NH}), 10.365(\mathrm{~s}, 1 \mathrm{H}, \mathrm{NH}) .{ }^{13} \mathrm{C}$ NMR $\left(100 \mathrm{MHz}, \mathrm{DMSO}-d_{6}\right) \delta_{\mathrm{C}}(\mathrm{ppm})$ : $23.17\left(\mathrm{Ar}_{-} \mathrm{CH}_{3}\right), 107.55,108.01,109.51,110.34,113.15,115.78,116.90,119.71,120.54,122.08$, 
124.54, 125.31, 126.01, 128.81, 129.20, 132.41, 135.70, 137.15, 139.22, 140.15, 141.77(Ar-C). MS $\left(\mathrm{M}^{+}\right)$: 460.08, Anal. Calcd. for $\mathrm{C}_{22} \mathrm{H}_{16} \mathrm{~N}_{6} \mathrm{O}_{2} \mathrm{~S}_{2}$ (460.53): C 57.38, H 3.50, N 18.25 Found: C 57.46, H 3.62, N 17.90\%.

$\boldsymbol{N}^{\mathbf{3}}$-(4-aminophenyl)-6-methyl- $\boldsymbol{N}^{\mathbf{2}}$-(4-(thiophen-2-yl)thiazol-2-yl)quinoxaline-2,3-diamine (4s) yellow solid, yield 79\%, m.p. $133-135^{\circ} \mathrm{C}$, IR (KBr, v, $\mathrm{cm}^{-1}$ ): 3313 (N-H Str.), 3250 (N-H Str.), 3033 (Ar C-H Str.), ${ }^{1} \mathrm{H}$ NMR (400 MHz, DMSO- $\left.d_{6}\right) \delta_{\mathrm{H}}(\mathrm{ppm}): \delta 2.365$ (s, 3H, $\left.\mathrm{Ar}-\mathrm{CH}_{3}\right), 7.005-8.100(\mathrm{~m}$, $11 \mathrm{H}, \mathrm{Ar}-\mathrm{H}), 10.125$ (s, 1H, NH), $10.200(\mathrm{~s}, 1 \mathrm{H}, \mathrm{NH}), 10.600\left(\mathrm{~s}, 1 \mathrm{H}, \mathrm{NH}_{2}\right) .{ }^{13} \mathrm{C} \mathrm{NMR}(100 \mathrm{MHz}$, DMSO- $\left.d_{6}\right) \delta_{\mathrm{C}}(\mathrm{ppm}): 26.34\left(\mathrm{Ar}-\mathrm{CH}_{3}\right), 113.21,114.26,115.67,117.18,118.90,121.40,123.64$, 125.28, 126.40, 128.03, 130.69, 131.15, 132.40, 135.16, 137.14, 138.90, 141.24, 142.33,144.00, 145.02, 146.21(Ar-C). $\mathrm{MS}\left(\mathrm{M}^{+}\right)$: 430.10, Anal. Calcd. for $\mathrm{C}_{22} \mathrm{H}_{18} \mathrm{~N}_{6} \mathrm{~S}_{2}$ (430.55): C 61.37, H 4.21, N 19.52 Found: C 61.48, H 4.55, N 19.20\%.

\section{6-methyl- $\boldsymbol{N}^{\mathbf{2}}$-(4-(thiophen-2-yl)thiazol-2-yl)- $\boldsymbol{N}^{\mathbf{3}}$-m-tolylquinoxaline-2,3-diamine (4t)}

yellow solid, yield 73\%, m.p. 140-142 C, IR (KBr, v, $\mathrm{cm}^{-1}$ ): 3286 (N-H Str.), 3241 (N-H Str.), 3018 (Ar C-H Str.), ${ }^{1} \mathrm{H}$ NMR (400 MHz, DMSO- $\left.d_{6}\right) \delta_{\mathrm{H}}(\mathrm{ppm}): \delta 2.300$ (s, 3H, Ar-CH $), 2.456$ (s, 3H, Ar$\left.\mathrm{CH}_{3}\right), 7.00-8.123(\mathrm{~m}, 11 \mathrm{H}, \mathrm{Ar}-\mathrm{H}), 10.656(\mathrm{~s}, 1 \mathrm{H}, \mathrm{NH}), 10.700(\mathrm{~s}, 1 \mathrm{H}, \mathrm{NH}) .{ }^{13} \mathrm{C} \mathrm{NMR}(100 \mathrm{MHz}$, DMSO- $\left.d_{6}\right) \delta_{\mathrm{C}}(\mathrm{ppm}): 24.19\left(\mathrm{Ar}-\mathrm{CH}_{3}\right), 27.18\left(\mathrm{Ar}-\mathrm{CH}_{3}\right), 112.20,113.21,114.66,117.12,119.10$, 122.42 , 124.10, 125.66, 126.14, 128.70, 130.1, 131.1, 132.63, 135.34, 137.18, 138.10, 141.39, 142.73,143.01, 144.01, 145.22(Ar-C). $\mathrm{MS}\left(\mathrm{M}^{+}\right)$: 429.11, Anal. Calcd. for $\mathrm{C}_{23} \mathrm{H}_{19} \mathrm{~N}_{5} \mathrm{~S}_{2}$ (429.56): C 64.31, H 4.46, N 16.30 Found: C 64.66, H 4.30, N 16.18\%.

\section{CONCLUSION}

In this article, we report the synthesis of three structurally correlated heterocyclic candidates, i.e., quinoxaline, thiophene and thiazole derivatives. The engaged synthetic strategy allows the construction of relatively complicated nitrogen and oxygen carrying heterocyclic system as well as the introduction of various aromatic substitutions into 3-position of quinoxaline system.

\section{Acknowledgments}

The authors are thankful to the Head, Department of Chemistry, Saurashtra University, Rajkot for providing ${ }^{1} \mathrm{H}$ NMR,${ }^{13} \mathrm{C}$ NMR, mass and FT-IR spectroscopy and research facilities.

\section{Reference}

[1] Perumal R. V., et al., Bioorg Med Chem Lett. 16 (2006) 2769.

[2] Hassan S. Y., Khattab S. N., Bekhit A. A., Bioorg Med Chem Lett. 16 (2006) 1753.

[3] Zhao Z., Leister W. H., Mahesh G. D., Bioorg Med Chem Lett. 15 (2005) 905.

[4] Arther G., Elor K. B., Robert G. S., Guo Z. Z., Richard J. P., Stanley D., John R. K., J Med Chem. 48 (2005) 744.

[5] Andres J., Belen Z., Ibnacio A., Antonio M., J Med Chem. 48 (2005) 2019.

[6] Ahmed A. R., Mehta L. K., Tetrahendron. 51 (1995) 12899.

[7] Sarges R., Howard H. R., Browne R. C., Label L. A., Seymour P. A., J Med Chem. 33 (1990) 2240 .

[8] Matsuoka M., Iwamato A., Furukawa N., J Heterocycl Chem. 29 (1992) 439.

[9] Nasr M. N. A., Arch Pharma Med Chem. 8 (2002) 389.

[10] Thomas K. R. J., Yu-Tai Tao and Chuen C. H., Lin J. T., J Master Chem. 12 (2002).

[11] Hirayama T., Yamasaki S., Hiroki A., Dyes Pigment. 67 (2005) 105. 
[12] Ldakov D., Anzenbacher P., Chem Comm. (2003) 1339.

[13] Torre M. H., Gambino D., de Certain A 1, Jeannette Araujo, Hugo Cerecetto, Mercedes González, María Laura Lavaggi, Amaya Azqueta, Antonio Monge Vega, Vega, Ulrich Abram and Antonio J Costa-Filho, Euro J Med Chem. 40 (2005) 473.

[14] Gazit A., Yeek K., Uecker A., Bioorg Med Chem. 11 (2003) 2007.

[15] Cheon H. G., Lee C. M., Kim B. T., Hwang K. J., Bioorg Med Chem Lett. 14 (2004) 1661.

[16] Refaat H. G., Moneeer A. A., Khalil O. M., Arch Pharma Res. 27 (2004) 1093.

[17] Bonde C., Norabeg J., Noer H., Zimmer J., Neurosci. 136 (2005) 779.

[18] Hui X., Desrivot J., Borier C., Philippe M. L., Xavier, F., Reynald H., Bruno F., Bioorg Med Chem Lett. 16 (2006) 815.

[19] Brown D. J., et al., Quinoxalines: Supplement II, The Chemistry of Heterocyclic Compounds; Wiley: New Jersey. (2004).

[20] Kim S. Y., Park K. H., Chung Y. K., Chem. Commun. (2005) 1321.

[21] Aparicio D., Attanasi O. A., Filippone P., Ignacio R., Lillini S., Mantellini F., Palacios F., Santos J. M., J. Org. Chem. 71 (2006) 5897.

[22] Wu Z., Ede N. J., Tetrahedron Lett. 42 (2001) 8115.

[23] Singh S. K., Gupta P., Duggineni S., Kundu, B., Synlett. (2003) 2147.

[24] Antoniotti S., Donach E., Tetrahedron Lett. 43 (2002) 3971.

[25] Mao L., Sakurai H., Hirao T., Synthesis. (2004) 2535.

[26] Zhao Z., Wisnoski D. D., Wolkenberg S. E., Leister W. H., Wang Y., Lindsley C. W., Tetrahedron Lett. 45 (2004) 4873.

[27] Bhosale R. S., Sarda S. R., Ardhapure S. S., Jadhav W. N., Bhusare S. R., Pawar R. P., Tetrahedron Lett. 46 (2005) 7183.

[28] More S. V., Sastry M. N. V., Wang C. C., Yao C. F., Tetrahedron Lett. 46 (2005) 6345.

[29] Islami M., Hassani Z., Arkivoc. 2008 (2008) 280.

[30] More S. V., Sastry M. N. V., Wang C. C., Tetrahedron Letters. 46 (2005) 6345.

[31] Heravi M. M., Taheri S., Bakhtiari, K., Oskooie H. A., Catalysis Communications. 8 (2007) 211.

[32] Kumar A., kumar S., Saxena A., Catalysis Communications. 9 (2008) 778.

[33] Cai J. J., Zou J. P., Pan X. Q., Zhang W., Tetrahedron Letters. 49 (2008) 7386.

[34] Huang T. K., Wang R., Shi L., Lu X. X., Catalysis Communications. 9 (2008) 1143.

[35] Dong F., Gong K., Fei Z., Zhou X., Liu Z., Catalysis Communications. 9 (2008) 317.

[36] Mirjalili B. B. F., Akbari A., Chinese Chemical Letters. 22 (2011) 753.

[37] Krishnakumar B., Velmurugan R., Jothivel S., Swaminathan M., Catalysis Communications.11 (2010) 997.

[38] Brown D. J., et al., The Chemistry of Heterocyclic Compounds, E. C. Taylor and P. Wipf, Eds., JohnWiley \& Sons, Hoboken, NJ, USA, 61 (2004) 1-510.

[39] Jafarpour M., Rezaeifard A., Danehchin M., Applied Catalysis A. 394 (2011) 48.

[40] Sharma R. K., Sharma C., Catalysis Communications. 12 (2011) 327.

[41] Sadjadi S., Sadjadi S., Hekmatshoar R., Ultrasonics Sonochemistry. 17 (2010) 764. 
[42] More S. V., Sastry M. N. V., Yao C. F., Green Chemistry. 8 (2006) 91.

[43] Rao K. T. V., Prasad P. S. S., Lingaiah N. S., Journal of Molecular Catalysis A. 312 (2009) 65.

[44] Niknam K., Saberi D., Mohagheghnejad M., Molecules. 14 (2009) 1915.

[45] Darabi H. R., Mohandessi S., Aghapoor K., Mohsenzadeh F., Catalysis Communications. 8 (2007) 389.

[46] Dodson R. M., King L. C., J Am Chem Soc. 67 (1945) 2242.

[47] Khunt H., Pipaliya P., Ghelani S., Naliapara Y. T., International Letters of Chemistry, Physics and Astronomy 24 (2014) 134.

[48] Patel A. S., Khunt H., Babariya J., Ghelani S., Naliapara Y. T., International Letters of Chemistry, Physics and Astronomy 30 (2014) 106.

[49] Gami P., Vilapara K., Khunt H., Babariya J., Naliapara Y. T., International Letters of Chemistry, Physics and Astronomy 30 (2014) 127.

[50] Khunt H., Babariya J., Dedakiya C., Naliapara Y. T., International Letters of Chemistry, Physics and Astronomy 30 (2014) 185.

[51] Khunt H., Babariya J., Naliapara Y. T., International Letters of Chemistry, Physics and Astronomy 31 (2014) 20.

[52] Prajapati D., Vilapara K., Naliapara Y. T., International Letters of Chemistry, Physics and Astronomy 33 (2014) 12. 